\title{
Collapsed 2-Dimensional Polymers on a Cylinder
}

\author{
Hsiao-Ping Hsu and Peter Grassberger \\ John-von-Neumann Institute for Computing, Forschungszentrum Jülich, D-52425 Jülich, Germany
}

(Dated: October 31, 2018)

\begin{abstract}
Single partially confined collapsed polymers are studied in two dimensions. They are described by self-avoiding random walks with nearest-neighbour attractions below the $\Theta$-point, on the surface of an infinitely long cylinder. For the simulations we employ the pruned-enriched-Rosenbluth method (PERM). The same model had previously been studied for free polymers (infinite lattice, no boundaries) and for polymers on finite lattices with periodic boundary conditions. We verify the previous estimates of bulk densities, bulk free energies, and surface tensions. We find that the free energy of a polymer with fixed length $N$ has, for $N \rightarrow \infty$, a minimum at a finite cylinder radius $R^{*}$ which diverges as $T \rightarrow T_{\theta}$. Furthermore, the surface tension vanishes roughly as $\left(T_{\theta}-T\right)^{\alpha}$ for $T \rightarrow T_{\theta}$ with $\alpha \approx 1.7$. The density in the interior of a globule scales as $\left(T_{\theta}-T\right)^{\beta}$ with $\beta \approx 0.32$.
\end{abstract}

Although the behaviour of polymers has been studied for many years, there are still a number of open questions. At high temperatures or in good solvents repulsive interactions (the excluded volume effect) dominate the conformation and the polymer chain tends to swell to a random coil. At low temperatures or in poor solvents attractive interactions between monomers dominate the conformation and the polymer chain tends to collapse and forms a compact dense globule. The coil-globule transition point is called the $\Theta$ point.

The behaviour in good solvents is accessible by field theory and thus well understood [1], e.g. the partition function scales as $Z \sim \mu^{N} N^{\gamma-1}$ at $T>T_{\theta}$. In poor solvents, much less is known exactly. For a long time it was e.g. widely believed that the partition sum scales in the same way, although already Lifshitz mean-field theory [2, 3] had predicted for $T<T_{\theta}$ that there should be also a surface term,

$$
Z(N, T) \sim a^{N} b^{-N^{s}} N^{\gamma-1},
$$

with $s=(d-1) / d$ and $b>1$. First numerical indications for this were given by [4, 5, 6], and theoretical arguments in favour of Eq. (11) were given in [7]. More recently, Eq. (1) was checked numerically in 8]. But it seems that there exists still no proof for Eq. (11), and it is not known how the surface tension (related to $b$ ) scales for $T \rightarrow T_{\theta}$. Moreover, the exponent $\gamma$ is not known [4, 6, 7], and it is not even clear whether it is universal in the collapsed phase.

In [8] we had studied two different geometries - globules on infinite lattices and polymers filling finite lattices with periodic boundary conditions - where surface tension should make very different contributions. In the present work we study a third geometry, namely polymers on the surface of an infinitely long cylinder, where the surface tension should make yet another contribution. Our main motivation was originally to have a simple geometry with straight "surfaces" so that the surface tension and its $T$-dependence can be more easily measured. But we found also another interesting effect: the bulk fugacity $\mu$ depends non-monotonically on the cylinder radius. It has a minimum at a finite radius $R^{*}$ which diverges for $T \rightarrow T_{\theta}$. Thus a collapsed polymer, when

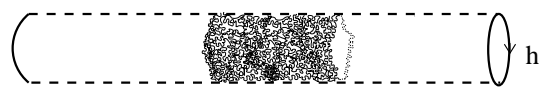

FIG. 1: Schematic drawing of a polymer chain growing on the surface of a cylinder.

wrapped around an elastic cylinder, will tend to squeeze it when $R>R^{*}$ and widen it when $R<R^{*}$.

A collapsed polymer on the surface of an infinitely long cylinder is modeled as an interacting self-avoiding random walk (ISAW) on a square lattice with periodic boundary conditions in the vertical direction, as shown in Fig. 1. The vertical lattice size is $h=2 \pi R$. The collapse is mediated by nearest neighbour attraction between monomers, described by a Boltzmann factor $q=e^{-\beta \epsilon}$ for each pair of non-bonded monomers occupying nearest neighbour sites on the lattice. Here $\beta=1 / k T$ and $\epsilon<0$ is the attractive potential. The partition sum is therefore

$$
Z=\sum_{\text {walks }} q^{m},
$$

where $m$ is the number of non-bonded occupied nearestneighbour pairs. We are interested in polymers which form collapsed globules, therefore $q>q_{\theta}$ with $q_{\theta}=$ $e^{-\epsilon / k T_{\theta}} \approx 1.95$ [10].

As argued in [2, 3] and verified in [4, 8], below the $\Theta$ point, the polymer forms a globule with uniform monomer density $\rho$ for large $N$ and $\rho \rightarrow 0$ as $T \rightarrow T_{\theta}$. Thus, the free energy for a collapsed polymer with chain length $N$ in infinite volume consists (except for a logarithmic term resulting from the last factor in Eq. (11)) of two parts: An extensive bulk contribution $\propto N$, and a surface contribution $\propto N^{(d-1) / d}$. For $d=2$, this reads (up to a factor $1 / T$ which will be suppressed in the following)

$$
-\ln Z_{N}(q) \approx \mu_{\infty}(q) N+\tilde{\sigma}(q) \sqrt{N}+O(\ln N)
$$

where $\mu_{\infty}$ is the chemical potential per monomer in an infinite chain, $\tilde{\sigma}$ is related to the surface tension (=free energy per unit of perimeter length) $\sigma$ and to the monomer 
density $\rho$ by

$$
\sigma=\frac{1}{2} \tilde{\sigma} \sqrt{\frac{\rho}{\pi}}
$$

The latter follows by assuming the globule to be a disk with radius $R_{g}$ fixed by $N=\pi R_{g}^{2} \rho$.

Surface terms are absent from the free energy if one has a finite $L \times L$ lattice with periodic boundary conditions, and if one chooses the polymer length such that the entire lattice is filled with finite density. More precisely, consider the quantity $\ln Z_{N}(q)+\mu N$. For $T<T_{\theta}$ this is non-convex, and for $\mu=\mu_{L}(q)$ it has two peaks of equal height, corresponding to the coexistence of two phases: One phase with very short chains and density $\approx 0$, and another phase with density $\rho_{L}(q)$. For $L \rightarrow \infty, \mu_{L}(q)$ and $\rho_{L}(q)$ tend monotonically [8] to $\mu_{\infty}(q)$ and $\rho$.

Finally, on the surface of an infinitely long cylinder with perimeter $h$, a collapsed polymer forms a cylindrical blob with length $L=\rho^{-1} N / h$ and surface free energy $2 \sigma h$,

$$
-\ln Z_{N}(q, h) \approx \mu_{h}(q) N+2 \sigma(q) h .
$$

Notice that the chemical potential per monomer will in general depend on $h$, even in the limit $N \rightarrow \infty$. For $h \rightarrow \infty$ we expect again to obtain the bulk limit,

$$
\mu_{h}(q) \rightarrow \mu_{\infty}(q) \quad h \rightarrow \infty .
$$

In principle we should also expect $\sigma(q)$ and $\rho(q)$ to depend on $h$. But we shall not study the detailed behaviour of $\rho$, and we shall neglect the $h$-dependence of $\sigma(q)$, since the surface term is a small correction anyhow. We also might expect logarithmic terms in $N$ to be present (just as also in the previous geometries), but they will also be neglected.

Our aim is to verify Eq. (5) numerically, to estimate the functions $\mu_{h}(q)$ and $\sigma(q)$, and to see whether they are compatible with the results for the other two geometries.

The pruned-enriched-Rosenbluth method (PERM) has been employed to study collapsed 2-d polymers in 8,10 . In [8] the predicted behaviour was verified numerically for globules on infinite lattices and for polymers filling finite lattices with periodic boundary conditions. More precisely, finite lattices were studied first, and from them estimates were obtained for $\mu_{\infty}$ and $\rho$ by extrapolation. Given these, subsequent simulations on infinite lattices gave rather precise estimates for $\sigma(q)$. But the latter was not done systematically for a wide range of temperatures, in particular the behaviour of $\sigma(q)$ for $q \rightarrow q_{\theta}$ was not studied. Thus we want to obtain also the latter in the present paper from cylinder geometry simulations.

For the first simulations, we choose $q=2.4$ which is deep inside the collapsed regime. From Eq. (5) we expect that plots of $\ln Z_{N}(q, h)+\mu N$ against $N$ should give horizontal lines for $N \rightarrow \infty$, if and only if $\mu=\mu_{h}(q)$. This gives estimates of $\mu_{h}(q)$. Using them, we plot in Fig. 2 $\ln Z_{N}(q, h)+\mu_{h}(q) N$ versus $N$ for various values of the

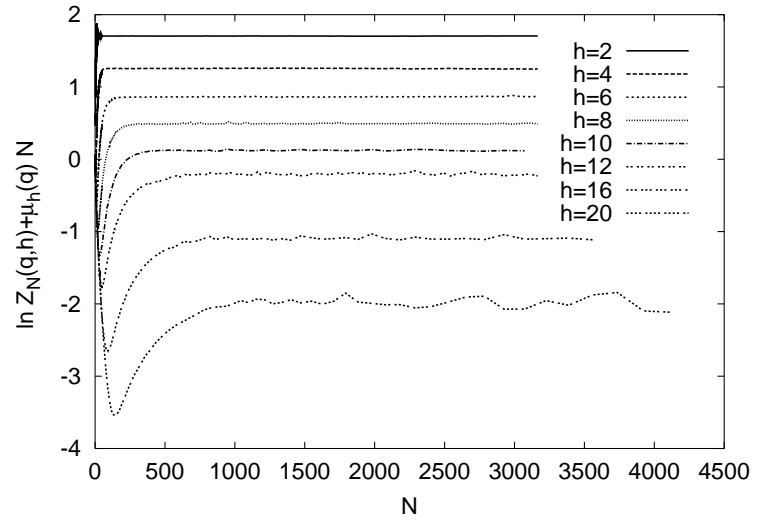

FIG. 2: $\ln Z_{N}(q, h)+\mu_{h}(q) N$ versus $N$ for $q=2.4$ and for various values of the cylinder circumference $h$. The values of $\mu$ were determined by demanding that these curves become horizontal as $N \rightarrow \infty$. The peaks seen in the curves for $h=16$ and 20 are statistical fluctuations.

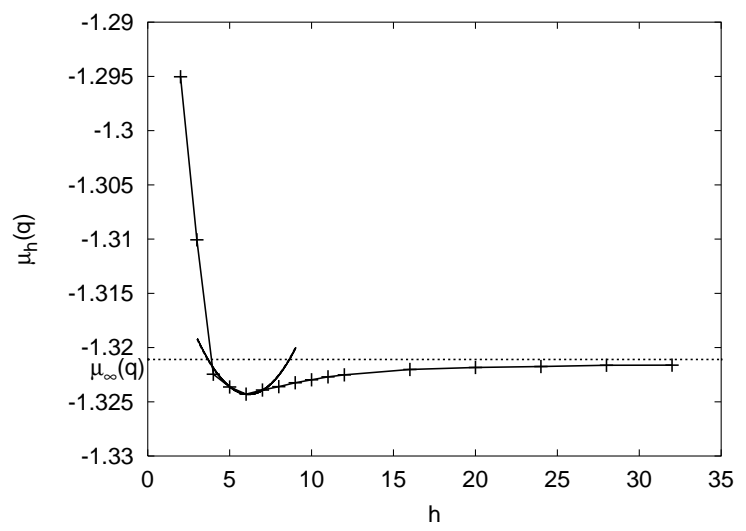

FIG. 3: $\mu_{h}(q=2.4)$ as obtained from Fig. 2, plotted against $h$. There is a minimum at $h=6.13 \pm 0.01$ which is determined by fitting a parabola (dashed curve) going through the three lowest points (which are at $h=5,6$, and 7). The dotted line is the asymptote $\mu_{\infty}(q)=-1.3211(1)$ approached for $h \rightarrow \infty$.

cylinder circumference $h$. For small $h$ we find perfect agreement with our expectations. For larger $h$ we see that PERM has problems in sampling long chains correctly, as should be expected. Fluctuations similar to those seen in the curve for $h=16$ prevent us from going to much larger $h$, except for $q$ close to $q_{\theta}$ where the efficiency of PERM increases.

Values of $\mu_{h}(q)$, again for $q=2.4$, are plotted in Fig. 3. For large $h$, we obtain $\mu_{\infty}(q)=-1.3211(1)$ in perfect agreement with [8]. But, surprisingly, the dependence of $\mu_{h}(q)$ on $h$ is not monotonic. Instead, we see a minimum at a finite value of $h$. In our simulations $h$ is an integer, but we can interpolate to continuous values by fitting a parabola, $\mu_{h}(q)=\mu_{\min }+b\left(h-h^{*}\right)^{2}$, through the three lowest points, as shown in Fig. 3. Such a fit gives $h^{*}(q=$ $2.4)=6.13(1)$ and $\mu_{\min }(q=2.4)=-1.3243(1)$. 


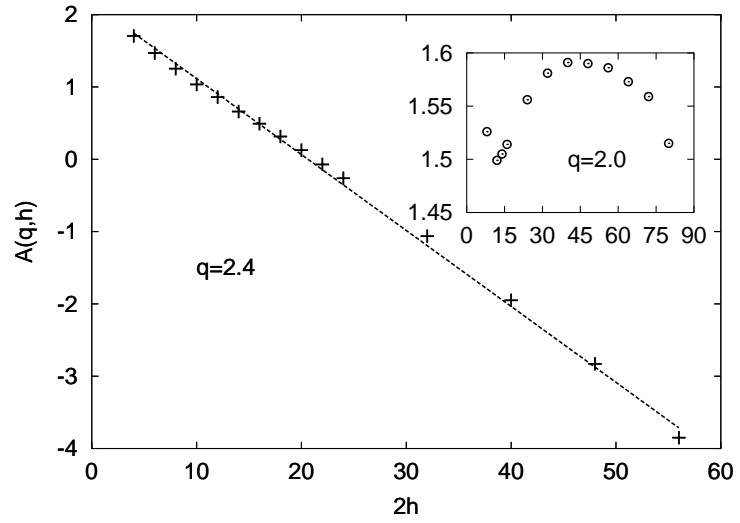

FIG. 4: $A(q, h)$ versus $2 h$ for $q=2.0$ and 2.4. The dashed line has slope $-\sigma=-0.105$. The points for $h=16$ and $h=20$ have large errors for $q=2.4$, as also seen from Fig. 2. Results for $q=2.0$ (insert) show the difficulty of measuring surface tension near $\Theta$-point.

Finally, in order to obtain the surface tension, we plot in Fig. 4

$$
A(q, h)=\lim _{N \rightarrow \infty}\left[\ln Z_{N}(q, h)+\mu_{h}(q) N\right]
$$

against $2 h$. The limit is of course obtained by using the parts of the curves in Fig. 2 where they are horizontal. From Eq. (5) we expect this to give a straight line with slope $-\sigma$. The result shows that $\sigma=0.105(5)$ at $q=2.40$. Actually, the line is slightly curved, showing that systematic errors are not negligible. For large $h$ they seem to arise from insufficient sampling. For small $h$ they result from the neglected terms discussed above. The present estimate should be compared to the estimate $\sigma=0.119(5)$ from [8]. Although the discrepancy is about 2 standard deviations, we consider this as reasonable agreement. Results for $q=2.0$ are also shown in Fig. 1, and illustrate the large corrections near the $\Theta$-point.

Analogous simulations as shown in Fig. 2 were also done at other values of $q$. The lengths $N$ depended strongly on $q$. Close to the $\Theta$-point the algorithm is very efficient and we obtained reliable high statistics data for $N$ up to 15,000 . The more the polymer is collapsed, the more difficult are the simulations. We stopped at $q=2.4$. As seen from Fig. 2, the errors decrease when $h$ becomes small, most likely because the walk is effectively Markovian with memory $\sim h^{2}$, and PERM is most efficient when memory is short. In all those simulations the results were similar. $\mu_{h}(q)$ had a minimum at a finite $h^{*}(q)$, and $A(q, h)$ versus $h$ was roughly linear with a negative slope. Results of $h^{*}(q), \mu_{\min }(q), \rho(q)$ and $\sigma(q)$ for a wide range of $q$ are shown in Fig. 5. 8 .

In Fig. 5 we can see that $h^{*}(q)$ increases as $q$ decreases. This is to be expected since the $\Theta$ transition is continuous, with a diverging correlation length for $T \rightarrow T_{\theta}$. Apart from the lattice constant (which is set to 1 here),

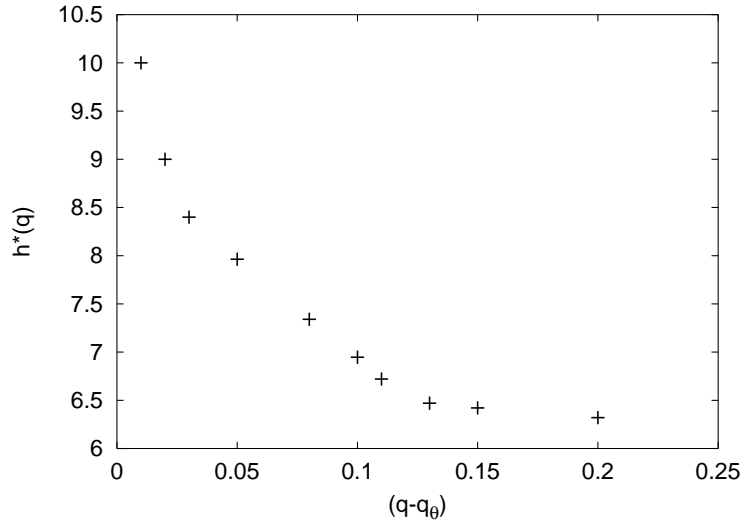

FIG. 5: "Optimal" cylinder circumference $h^{*}(q)$ against $\left(q-q_{\theta}\right)$. At $h=h^{*}(q)$, the chemical potential is minimal, $\mu_{h^{*}}(q)=\mu_{\min }(q)$.

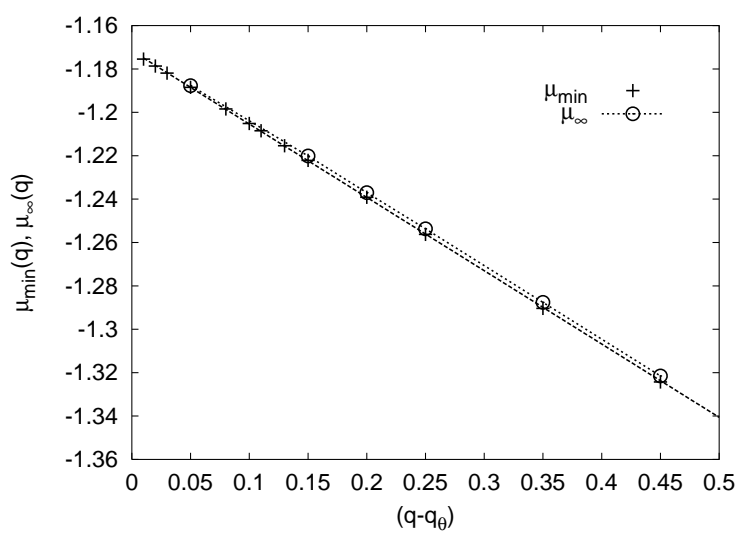

FIG. 6: $\mu_{\min }$ and $\mu_{\infty}$ plotted against $\left(q-q_{\theta}\right)$ for various values of $q$. The slope of the straight line is roughly equal -0.34 . $\mu_{\infty}$ were obtained from extrapolation to large $h$.

the correlation length is the only relevant length scale. The divergence of $h^{*}(q)$ when approaching the $\Theta$ point thus indicates that the existence of the minimum is not a lattice artifact and is presumably a genuine feature of the continuum theory. The simulations are not precise enough to allow a meaningful fit to a power law and a determination of a critical exponent. This exponent would also be effected strongly by the uncertainty of $T_{\theta}$. To reduce the latter to a minimum, we performed extensive simulations of free (i.e. infinite lattice) ISAWs near the estimated $\Theta$ point, with the result $q_{\theta}=1.9487(5)$ which is in good agreement with the best previous estimate [10].

In Fig. 6 we plot $\mu_{\min }(q)$ and $\mu_{\infty}(q)$ versus $\left(q-q_{\theta}\right)$. We see that both decrease linearly with temperature. The difference between them depends weakly on the temperature. It seems to vanish for $T \rightarrow T_{\theta}$.

The monomer density $\rho$ inside the "globule" is constant for $N \gg h^{2} \gg 1$ and fixed temperature. It can be measured by measuring the $x$-coordinate (parallel to the 


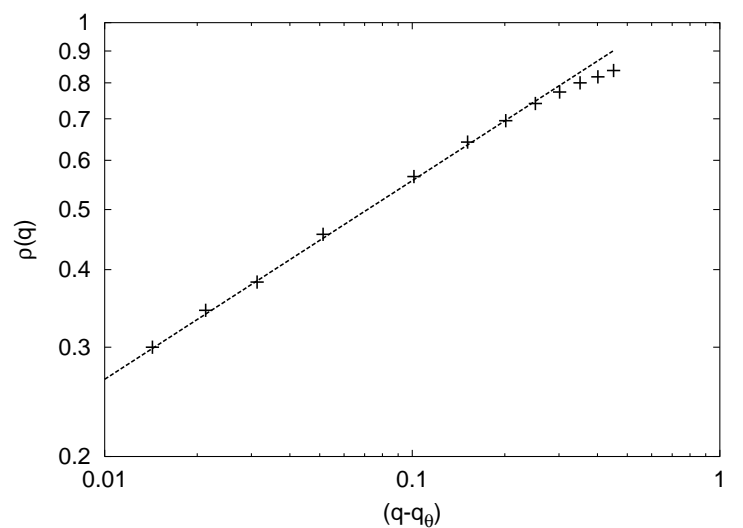

FIG. 7: Log-log plot of $\rho$, as obtained from the growth of $\left\langle\left|x_{N}\right|\right\rangle$ with $N$ for large $N$ and $h$, against $\left(q-q_{\theta}\right)$. For this plot we used $q_{\theta}=1.9487$. The dashed line is $\rho \propto\left(q-q_{\theta}\right)^{0.32}$.

cylinder) of the end point of the chain. Since the chain has to grow either into the positive or into the negative direction, we have

$$
\frac{d\left\langle\left|x_{N}\right|\right\rangle}{d N} \sim(h \rho)^{-1}, \quad N \rightarrow \infty .
$$

For small values of $h$ there are finite size corrections to the density, but it is easy to extrapolate to $h \rightarrow \infty$. The resulting densities are plotted versus $q$ in Fig. 7. They show a power law

$$
\rho \sim\left(q-q_{\theta}\right)^{\beta}
$$

with $\beta=0.32 \pm 0.02$. We are not aware of previous estimates of $\beta$, either theoretically or by simulations.

The values of the surface tension $\sigma(q)$ obtained by the same way as shown in Fig. 1 are shown in Fig. 8. They follow a power law, $\sigma \sim\left(q-q_{\theta}\right)^{\alpha}$, with $\alpha \approx 1.7 \pm 0.1$. For the parameter $\tilde{\sigma}$ this implies that

$$
\tilde{\sigma} \sim\left(q-q_{\theta}\right)^{\alpha-\beta / 2} \sim\left(q-q_{\theta}\right)^{1.54 \pm 0.1} .
$$

This is incompatible with the theoretical prediction $\tilde{\sigma} \sim$ $\left(T_{\theta}-T\right)^{7 / 6}$ quoted in [6].
In summary, we have studied collapsed polymers on the surface of a cylinder. Using the PERM algorithm we could simulate chains of lengths between 3000 (far below $T_{\theta}$ ) and $10^{4}$ (near $T_{\theta}$ ) for a wide range of temperatures. We have verified that the concept of a surface tension applies to 2D collapsed polymers, and have determined its scaling near the $\Theta$ point. We also determined the scaling of the monomer density in the collapsed phase, as $T \rightarrow T_{\theta}$. In addition, we found a surprising nonmonotonic dependence of the chemical potential on the perimeter of the cylinder. It shows that for any temperature there is a special value of the perimeter where the free energy of the polymer is minimal. The fact that this value diverges when the $\Theta$-temperature is approached suggests that this is not an artifact of the square lattice but a generic feature.

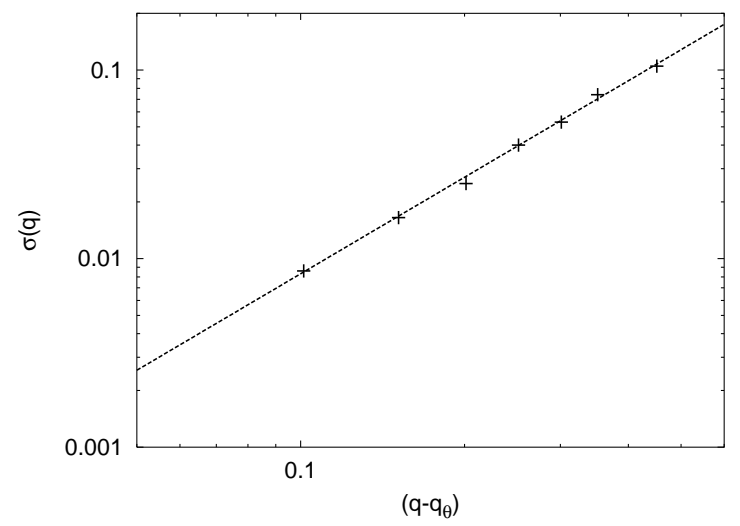

FIG. 8: Log-log plot of $\sigma$, as obtained from Fig. \& against $\left(q-q_{\theta}\right)$. For this plot we used $q_{\theta}=1.9487$. The dashed line is $\sigma \propto\left(q-q_{\theta}\right)^{1.7}$.

Acknowledgements: We thank Dr. Walter Nadler for valuable discussions. Parts of the simulations were done on a PC-farm in Taipei. H. P. thanks the Computing Centre of Academia Sinica in Taipei, Taiwan for providing the computing facilities.
[1] P. G. de Gennes, Scaling Concepts in Polymer Physics (Cornell University Press, Ithaca, 1979).

[2] A. Yu. Grosberg and D. V. Kuznetsov, Macromolecules 25, 1970 (1992).

[3] I. M. Lifshitz, JETP 28, 1280 (1968).

[4] A. L. Owczarek, T. Prellberg, and R. Brak, Phys. Rev. Lett. 70, 951 (1993).

[5] P. Grassberger and R. Hegger, J. Chem. Phys. 102, 6881 (1995).
[6] P.P. Nidras, J. Phys. A 29, 7929 (1996).

[7] B. Duplantier, Phys. Rev. Lett. 71, 4274 (1993).

[8] P. Grassberger and H.-P. Hsu, Phys. Rev. E 65, 031807 (2002).

[9] P. Grassberger, Phys. Rev. E 56, 3682 (1997).

[10] G.T. Barkema, U. Bastolla, and P. Grassberger, J. Stat. Phys. 90, 1311 (1998). 